\title{
Genetic divergence among landraces and improved common bean genotypes in the central-southern region of Mato Grosso state in Brazil
}

\author{
P.R.J. dos Santos ${ }^{1}$, M.A.A. Barelli ${ }^{2}$, R. Felipin-Azevedo ${ }^{1}$, V.P. da Silva ${ }^{3}$, \\ T.A.S. Gilio ${ }^{1}$, T.C. de Oliveira ${ }^{3}$, D.L. Gonçalves ${ }^{1}$ and J.P. Poletine ${ }^{4}$ \\ ${ }^{1}$ Programa de Pós-Graduação em Genética e Melhoramento de Plantas, \\ Universidade do Estado de Mato Grosso, Cáceres, MT, Brasil \\ ${ }^{2}$ Departamento de Agronomia, Universidade do Estado de Mato Grosso, \\ Cáceres, MT, Brasil \\ ${ }^{3}$ Programa de Pós-Graduação em Biodiversidade Amazônica e \\ Biotecnologia, Rede Bionorte Universidade do Estado de Mato Grosso, \\ Cáceres, MT, Brasil \\ ${ }^{4}$ Departamento de Ciências Agronômicas, Universidade Estadual de \\ Maringá, Campus de Umuarama, Umuarama, PR, Brasil \\ Corresponding author: R. Felipin-Azevedo \\ E-mail: rafhaelfelipin@gmail.com \\ Genet. Mol. Res. 18 (2): gmr18299 \\ Received March 12, 2019 \\ Accepted May 27, 2019 \\ Published June 30, 2019 \\ DOI http://dx.doi.org/10.4238/gmr18299
}

ABSTRACT. The common bean is widely distributed throughout Brazil and within Mato Grosso state. It has wide genetic variability and adaptability to various environmental conditions. We evaluated the genetic divergence and agronomic performance of common bean genotypes in order to initiate a bean breeding program in the region of Cáceres, MT. We included 58 genotypes (40 traditional accessions, 14 lines and four cultivars) using a randomized block design with three replications, at Empresa de Pesquisa, Assistência e Extensão Rural do Mato Grosso, evaluating 10 morphological and agronomic characteristics. The data were submitted to analysis of 
variance and cluster analysis based on Mahalanobis distance; we used the Tocher and UPGMA grouping methods to group to the genotypes. Through analysis of variance, we detected significant differences at $1 \%$ probability for all traits. The shortest genetic distance was found between genotypes 13 and $26\left(D_{i i^{\prime}}^{2}=1.51\right)$ as the most similar, while 20 and $4\left(D_{i i^{\prime}}^{2}=346.00\right)$ were the most dissimilar. The Tocher and UPGMA clustering methods were similar in grouping the genotypes, with the most divergence in different groups. Crossing of the earliest genotypes $(11,13$ and 16) with the improved genotypes (43 and 51) resulted in the greatest heterotic effect, increasing the likelihood of the emergence of superior genotypes in the progenies, which is beneficial for the initiation of the bean breeding program for the Cerrado region of Cáceres in Mato Grosso.

Key words: Phaseolus vulgaris; Plant breeding; Genetic divergence

\section{INTRODUCTION}

The common bean (Phaseolus vulgaris) is a legume of great nation-wide importance, cultivated in all Brazilian states and with three seasons distributed throughout the year (Vieira, 2006). It is important for human consumption, as it is a source of carbohydrates, proteins, fibers, minerals and vitamins for populations around the world (Broughton et al., 2003; Beraldo et al., 2009; Tsutsumi et al., 2015).

In the world scenario, Brazil ranks as the third largest producer, being surpassed only by Myanmar and India (FAO, 2019); the states with the highest production are Paraná, Minas Gerais, Mato Grosso, Bahia and Goiás (CONAB , 2019). The state of Mato Grosso is one of the largest producers of common bean in Brazil; according to CONAB (2019), it is the third largest producer, especially Sorriso county, which is the largest producer of the country. In addition, the center-south region of the state has gained prominence in relation to the cultivation of common bean recently, mainly common beans of the carioca line.

The common bean crop in Brazil is extremely diverse due to different environments and diseases, multiple production systems and farmer preferences (Coelho et al., 2007; Bertoldo et al., 2014). Also, Brazil can be considerate a secondary diversity center (Burle et al., 2010). The success of a breeding program depends on the genetic diversity available to breeders (Hoisington et al., 1999) for developing genotypes highly adapted to distinct environments and production systems. The importance of plant breeding will likely increase in the future in an effort to feed a growing population demanding specific characteristics; there is also a need to reduce environmental effects due to the use of agricultural inputs and climate change, specifically those associated with increases in temperature and water stress. The available evidence, particularly the degree of genetic diversity of common bean, suggests an opportunity for successful breeding programs (Ramalho et al., 2009).

Furthermore, the characterization of the genetic variability of local cultivars together with the improved ones is of great importance for plant breeding programs, because it provides information about the variability existing in these materials, taking into 
account the importance that these have due to their adaptation to the environmental conditions of a particular region; it allows the identification of genotypes to compose breeding programs, with the objective of using this local adaptation in crosses with improved cultivars, aiming at obtaining superior genotypes (Nass et al., 2012).

According to Ferreira Junior et al. (2015), genetic variability information to choose parental for hybridization should be accompanied by information regarding the performance of the genotypes regarding production components, precocity and interesting characteristics for mechanized harvesting.

In this sense, studies of genetic variability are fundamental for the breeding of plants, since they provide information about breeders that allow, when crossed, a great heterotic effect and greater segregation in recombinants, increasing the probability of the appearance of superior genotypes in the progenies (Silva et al., 2008). According to Alvares et al. (2012) to generate segregating populations for selection, the genetic complementation of the parents should be positive, so the grouping techniques help in guiding the choice of parents or genitors.

We evaluated the genetic divergence and agronomic performance of common bean genotypes in order to direct the beginning of a common bean breeding program in Mato Grosso state, Brazil.

\section{MATERIAL AND METHODS}

The experiment was conducted in an experimental area belonging to the Government Company, Empresa de Pesquisa, Assistência e Extensão Rural do Mato Grosso (Empaer - MT), in Cáceres county, MT. 58 common bean genotypes were evaluated, among which 40 were traditional genotypes cultivated in the central south region of Mato Grosso State, 14 advanced lines and from commercial cultivars from the Phaseolus germplasm Active Bank (BAG) of the State University of Mato Grosso UNEMAT, Cáceres campus (Table 1).

The typical weather of the region, according to the classification of Köppen, is tropical, hot, humid and dry winter (Awa), with a rainy season from October to March, and drought from April to September (Dallacort et al., 2014). The soil is classified as Chernosolic Eutrophic Yellow Red Argissolo, with a medium clay texture (Arantes et al., 2012).

Seeding was carried out in the second planting season (dry bean, cultivated in the summer-fall), adopting a planting density of 20 plants per linear meter, with subsequent thinning after emergence, leaving 15 plants per linear meter of initial stand. In addition to plowing and harvesting, fertilization was carried out at depths of $0-10$ and $10-20 \mathrm{~cm}$, based on soil chemical, to reach the nutritional requirements of the crop.

The cultural treatments were those recommended for common bean crop, in addition to irrigation whenever necessary, aiming at maintaining the amount of water in the soil profile. Harvesting was performed respecting the physiological maturity period (when $90 \%$ of the whole pods were dry) drying the grains in the sun until reaching $12 \%$ of humidity. 
Table 1. Common bean genotypes that were evaluated, including commercial group, flower color, gene pool and origin (Cáceres, MT).

\begin{tabular}{|c|c|c|c|c|c|}
\hline & Name & Commercial Group & Flower Color & Gene pool & Origin \\
\hline 1 & BG-UNEMAT -18 & Manteigão & White & Mesoamerican & UNEMAT \\
\hline 2 & BG-UNEMAT - 28 & Manteigão & White & Mesoamerican & UNEMAT \\
\hline 3 & BG-UNEMAT - 11 & Bolinha & White & Andean & UNEMAT \\
\hline 4 & BG-UNEMAT - 27 & Mulatinho & White & Mesoamerican & UNEMAT \\
\hline 5 & BG-UNEMAT - 22 & Carioca & White & Mesoamerican & UNEMAT \\
\hline 6 & BG-UNEMAT - 40 & Carioca & White & Mesoamerican & UNEMAT \\
\hline 7 & BG-UNEMAT - 17 & Preto & Violet & Mesoamerican & UNEMAT \\
\hline 8 & BG-UNEMAT - 32 & Manteigão & White & Mesoamerican & UNEMAT \\
\hline 9 & BG-UNEMAT - 4 & Mulatinho & White & Mesoamerican & UNEMAT \\
\hline 10 & BG-UNEMAT - 30 & Roxo & White & Mesoamerican & UNEMAT \\
\hline 11 & BG-UNEMAT - 3 & Manteigão & White & Mesoamerican & UNEMAT \\
\hline 12 & BG-UNEMAT - 6 & Carioca & White & Mesoamerican & UNEMAT \\
\hline 13 & BG-UNEMAT - 29 & Mulatinho & White & Mesoamerican & UNEMAT \\
\hline 14 & BG-UNEMAT - 35 & Manteigão & White & Mesoamerican & UNEMAT \\
\hline 15 & BG-UNEMAT - 42 & Carioca & White & Mesoamerican & UNEMAT \\
\hline 16 & BG-UNEMAT - 23 & Carioca & White & Mesoamerican & UNEMAT \\
\hline 17 & BG-UNEMAT - 26 & Mulatinho & White & Mesoamerican & UNEMAT \\
\hline 18 & BG-UNEMAT - 31 & Mulatinho & White & Mesoamerican & UNEMAT \\
\hline 19 & BG-UNEMAT - 37 & Roxo & White & Mesoamerican & UNEMAT \\
\hline 20 & BG-UNEMAT - 38 & Manteigão & Violet & Andean & UNEMAT \\
\hline 21 & BG-UNEMAT - 75 & Carioca & White & Mesoamerican & UNEMAT \\
\hline 22 & BG-UNEMAT - 1 & Carioca & White & Mesoamerican & UNEMAT \\
\hline 23 & BG-UNEMAT - 56 & Mulatinho & White & Mesoamerican & UNEMAT \\
\hline 24 & BG-UNEMAT - 50 & Carioca & White & Mesoamerican & UNEMAT \\
\hline 25 & BG-UNEMAT - 49 & Carioca & White & Mesoamerican & UNEMAT \\
\hline 26 & BG-UNEMAT - 45 & Manteigão & White & Mesoamerican & UNEMAT \\
\hline 27 & BG-UNEMAT - 46 & Carioca & White & Mesoamerican & UNEMAT \\
\hline 28 & BG-UNEMAT - 72 & Manteigão & White & Mesoamerican & UNEMAT \\
\hline 29 & BG-UNEMAT - 55 & Carioca & White & Mesoamerican & UNEMAT \\
\hline 30 & BG-UNEMAT - 16 & Manteigão & White & Mesoamerican & UNEMAT \\
\hline 31 & BG-UNEMAT - 60 & Manteigão & White & Mesoamerican & UNEMAT \\
\hline 32 & BG-UNEMAT - 58 & Manteigão & White & Mesoamerican & UNEMAT \\
\hline 33 & BG-UNEMAT - 5 & Carioca & Violet & Mesoamerican & UNEMAT \\
\hline 34 & BG-UNEMAT - 68 & Bolinha & White & Mesoamerican & UNEMAT \\
\hline 35 & BG-UNEMAT - 7 & Manteigão & White & Andean & UNEMAT \\
\hline 36 & BG-UNEMAT - 47 & Roxo & White & Mesoamerican & UNEMAT \\
\hline 37 & BG-UNEMAT - 12 & Carioca & White & Mesoamerican & UNEMAT \\
\hline 38 & BG-UNEMAT - 13 & Carioca & White & Mesoamerican & UNEMAT \\
\hline 39 & BG-UNEMAT - 20 & Carioca & White & Mesoamerican & UNEMAT \\
\hline 40 & BG-UNEMAT - 21 & Carioca & White & Mesoamerican & UNEMAT \\
\hline 41 & Pérola & Carioca & White & Mesoamerican & IAPAR \\
\hline 42 & IPR Campos gerais & Carioca & White & Mesoamerican & IAPAR \\
\hline 43 & IPR Uirapuru & Preto & Violet & Mesoamerican & IAPAR \\
\hline 44 & CNFP 10104 & Preto & Violet & Mesoamerican & IAPAR \\
\hline 45 & C 4-7-7-2-2 & Carioca & White & Mesoamerican & IAC \\
\hline 46 & C4-7-8-1-2 & Carioca & White & Mesoamerican & IAC \\
\hline 47 & CHC 98-42 & Carioca & White & Mesoamerican & EPAGRI \\
\hline 48 & CHP 01-238 & Preto & Violet & Mesoamerican & EPAGRI \\
\hline 49 & CNFC 10762 & Carioca & White & Mesoamerican & CNPAF \\
\hline 50 & CNFP 10794 & Preto & Violet & Mesoamerican & CNPAF \\
\hline 51 & FT 08-47 & Carioca & White & Mesoamerican & FT \\
\hline 52 & FT 08-75 & Carioca & White & Mesoamerican & FT \\
\hline 53 & LEC 01-11 & Carioca & White & Mesoamerican & UEM \\
\hline 54 & LEP 02-11 & Preto & Violet & Mesoamerican & UEM \\
\hline 55 & LP 09-192 & Preto & Violet & Mesoamerican & IAPAR \\
\hline 56 & LP 09-40 & Carioca & White & Mesoamerican & IAPAR \\
\hline 57 & TB02-23 & Preto & Violet & Mesoamerican & CPACT \\
\hline 58 & TB $03-13$ & Enxofre & Violet & Andean & CPACT \\
\hline
\end{tabular}


The agronomic characteristics evaluated were: a) Number of days till flowering (FLOWER): period from emergence to full opening of the first flower, in 50\% of the plants of the plot, in days; b) Average height of first pod insertion (AHFPI): in centimeters (cm), measurement from the soil base until insertion of the first pod of 10 plants per plot; c) Final average height of plants (FAHP): measurement in centimeters from ground level to the end of 10 plants per plot; d) Average longitudinal length of pods (ALLP): in centimeters, longitudinal measurement from one end to the other of the pod, ten pods of the 10 plants evaluated per plot; e) Average number of pods per plant (ANPP): in 10 plants evaluated in each plot; f) Average number of seeds per pod (ANSP): in 10 pods of the ten plants evaluated per plot; g) Average number of seeds per plant (ANSPL): in 10 evaluated plants of each plot; h) average weight of 100 seeds (W100): in grams (g) of four samples of 100 seeds of each plot, with humidity of $12 \%$; i) Cycle (CYCLE): number of days of emergency until the harvest season on each plot; j) Grain Productivity (GPROD): in kg per hectare $\left(\mathrm{kg} . \mathrm{ha}^{-1}\right)$, relation between the total weight of the grains of each plot and the respective number of plants.

The obtained data were submitted to analysis of variance, considering the effect of fixed genotype and the clustering test of the average employed was that of Skott Knott. For the estimation of the genetic divergence among the accesses, a multivariate analysis was used based on the generalized distance of Mahalanobis, then performing Tocher's cluster analysis (RAO, 1952) and of Average Clustering Between Groups (UPGMA), by the computational software Genes (Cruz, 2013).

\section{RESULTS AND DISCUSSION}

Significant differences at $1 \%$ probability by the $\mathrm{F}$ test $(\mathrm{P}<0.01)$ were found for all traits (Table 2), confirming the existence of genetic variability among the genotypes. The coefficient of variation ranged from 2.07 and 24.39\%, for FLOWER and GPROD, respectively, indicating adequate experimental accuracy, being considered reliable, according to Cabral et al. (2011) when studying the genetic divergence of 57 common bean accessions (31 local genotypes, 20 Embrapa genotypes and six commercial cultivars), obtained a coefficient of variation between $2.70 \%$ and $20.86 \%$, for variables similar to those studied, considering this constant coefficient of variation to determine the variability among the genotypes.

Table 2. Significance of mean square and percentage coefficients of experimental variation for the 10 characteristics evaluated in 58 common bean genotypes.

\begin{tabular}{|c|c|c|c|c|c|c|c|c|c|c|c|}
\hline \multirow{2}{*}{$\begin{array}{l}\text { Source } \\
\text { Variation }\end{array}$} & \multirow{2}{*}{ DF } & \multicolumn{10}{|c|}{ Mean square } \\
\hline & & FLOWER & AHFPI & FAHP & ALLP & ANPP & ANSP & ANSPL & W100 & CYCLE & GPROD \\
\hline Block & 2 & 0.36 & 23.78 & 62.34 & 0.55 & 28.75 & 0.15 & 968.36 & 9.99 & 12.16 & $1.854 .461,75$ \\
\hline Genotype & 57 & $13.78^{* *}$ & $32.84 * *$ & $78.71 * *$ & $1.26 * *$ & $30.13^{* *}$ & $0.69 * *$ & $646.26^{* *}$ & $67.48^{* *}$ & $317.24 * *$ & $2.303 .699 .17 * *$ \\
\hline Residue & 114 & 0.60 & 9.24 & 14.16 & 0.22 & 11.08 & 0.25 & 265.82 & 2.28 & 11.51 & 532.609 .08 \\
\hline Average & & 37.29 & 21.22 & 51.86 & 10.33 & 17.95 & 5.38 & 70.72 & 25.31 & 84.98 & 2.991 .67 \\
\hline C.V. $(\%)$ & & 2.07 & 14.33 & 7.26 & 4.54 & 18.54 & 9.30 & 23.05 & 5.97 & 3.99 & 24.39 \\
\hline
\end{tabular}


Similar results were found by Barelli et al. (2009) analyzing nine agronomic characteristics of 35 traditional common bean cultivars in Mato Grosso do Sul state, found values of coefficient of variation from $0.80 \%$ to $24.41 \%$, considered adequate to study genetic divergence among cultivars, demonstrating good execution of the experiment. Table 3 shows the results obtained by the Scott-Knott cluster test for 10 characteristics of the 58 evaluated genotypes, where for the FLOWER characteristic, the flowering period ranged from 31 to 40 days, which made it possible to divide the genotypes into five distinct groups. The traditional accessions behaved as early as the majority of the lines and the improved cultivars evaluated, where the earliest genotypes were 3 and 57, with 31 days for flowering. These results resemble those obtained by Lima et al. (2012), evaluating 100 genotypes of common bean in Coimbra, Minas Gerais, in which the average flowering values were 34.4 to 43.3 days. It is also noted that this characteristic is key to evaluating precocity of the common bean crop (Silva et al., 2007), and the use of early flowering genotypes gives the farmer better use of the area and can optimize the cultural practices carried out in the crop and adjust its planting and harvesting schedule according to the environmental conditions of the growing region (Ribeiro et al., 2004).

For the CYCLE characteristic, five groups were formed, the latest belonging to Group I and the earliest to Group V, with a variation of 73 to 100.7 days, where the improved genotypes were mostly allocated to the latest group, and the traditional accessions all can carry out their productive cycle with less time than the improved genotypes.

Considering both FLOWER and CYCLE, genotype 3 is the most precocious compared to the other analyzed, the great majority of the traditional accessions that present a little later flowering but it is as early as the 3 for closing of the cycle, which makes it possible to say that as for the precocity characteristic, the traditional accessions present a set of favorable alleles for breeding for a shorter flowering period.

Results concordant with Buratto et al. (2007), where comparing days for flowering and days for physiological maturation, found from 28 to 43 days for flowering, followed by 54 to 88 days for physiological maturation, evidencing significant differences between promising lines and cultivars, observing that the cultivars presented less precocious than most of the lines studied for the two characteristics.

For the FAHP characteristic, four distinct groups were formed, ranging from 40.53 to $62.27 \mathrm{~cm}$. Salgado et al. (2011) evaluating the behavior of common bean genotypes in Tocantins, obtained plant height considered high between 75.11 to $108.90 \mathrm{~cm}$, values higher than those observed in this research. However, Oliveira et al. (2012) also evaluating common bean genotypes in Tocantins obtain similar results for plant height (ranging from 38.20 to $74.70 \mathrm{~cm}$ ) and considers the results of this characteristic for the mechanized harvest to be satisfactory for the cultivars.

In the AHFPI evaluation two distinct groups were formed, ranging between 16.4 and $29.07 \mathrm{~cm}$ of insertion of the first pod, which favors the health of pods and grains, and for ALLP, the genotypes were clustered into 3 distinct groups (ranging from 9.09 to 11.94 $\mathrm{cm})$. In this way the genotypes studied have characteristics of AHFPI and ALLP that favor the mechanized harvest, which is extremely positive for crop improvement. According to Peluzio et al. (2009), the ideal height of first pod insertion varies between 10.00 and 15.00 $\mathrm{cm}$, since the insertion of very low pods can lead to losses in the harvest and quality of the harvested grains, caused by fungal diseases of soil in pods, in addition to reach of demand for mechanized harvesting. 
Table 3. Clustering of the average of 58 common bean genotypes by the Scott-Knott method, estimated from 10 agronomic characteristics.

\begin{tabular}{|c|c|c|c|c|c|c|c|c|c|c|}
\hline Gen $^{1 /}$ & FLOWER & AHFPI & FAHP & ALLP & ANPP & ANSP & ANSPL & W100 & CYCLE & GPROD \\
\hline 1 & $36.33 \mathrm{c}$ & $16.53 \mathrm{~b}$ & $56.83 \mathrm{a}$ & $11.74 \mathrm{a}$ & $20.23 \mathrm{a}$ & $5.70 \mathrm{a}$ & $77.80 \mathrm{a}$ & $25.91 \mathrm{e}$ & $78.0 \mathrm{e}$ & $2972.50 \mathrm{c}$ \\
\hline 2 & $35.00 \mathrm{c}$ & $16.77 \mathrm{~b}$ & $53.80 \mathrm{~b}$ & $9.92 \mathrm{c}$ & $19.93 \mathrm{a}$ & $5.03 \mathrm{~b}$ & $79.90 \mathrm{a}$ & $19.76 \mathrm{~g}$ & $74.3 \mathrm{e}$ & $2339.54 \mathrm{c}$ \\
\hline 3 & $31.00 \mathrm{e}$ & $17.37 \mathrm{~b}$ & $42.13 \mathrm{~d}$ & $10.25 \mathrm{c}$ & $16.00 \mathrm{~b}$ & $4.07 \mathrm{~b}$ & $44.90 \mathrm{~b}$ & $32.86 \mathrm{c}$ & $73.0 \mathrm{e}$ & $2524.29 \mathrm{c}$ \\
\hline 4 & $37.00 \mathrm{c}$ & $22.83 \mathrm{a}$ & $48.47 \mathrm{c}$ & $10.63 \mathrm{~b}$ & $19.57 \mathrm{a}$ & $5.53 \mathrm{a}$ & $75.21 \mathrm{~b}$ & $17.21 \mathrm{~g}$ & $73.0 \mathrm{e}$ & $1802.22 \mathrm{c}$ \\
\hline 5 & $39.00 \mathrm{a}$ & $22.40 \mathrm{a}$ & $47.03 \mathrm{c}$ & $10.04 \mathrm{c}$ & $15.13 \mathrm{~b}$ & $5.83 \mathrm{a}$ & $66.19 b$ & $24.72 \mathrm{e}$ & $87.7 \mathrm{c}$ & $2708.54 \mathrm{c}$ \\
\hline 6 & $39.00 \mathrm{a}$ & $23.27 \mathrm{a}$ & $53.60 \mathrm{~b}$ & $10.37 \mathrm{c}$ & $15.60 \mathrm{~b}$ & $5.20 \mathrm{~b}$ & $53.30 \mathrm{~b}$ & $26.76 \mathrm{e}$ & $88.7 \mathrm{~b}$ & $2211.49 \mathrm{c}$ \\
\hline 7 & $38.67 \mathrm{a}$ & $23.17 \mathrm{a}$ & $61.70 \mathrm{a}$ & $9.67 \mathrm{c}$ & $17.17 \mathrm{~b}$ & $5.53 \mathrm{a}$ & $75.93 \mathrm{~b}$ & $23.89 \mathrm{e}$ & $87.7 \mathrm{c}$ & $2845.84 \mathrm{c}$ \\
\hline 8 & $36.67 \mathrm{c}$ & $18.07 \mathrm{~b}$ & $50.53 \mathrm{c}$ & $11.83 \mathrm{a}$ & $20.77 \mathrm{a}$ & $5.27 \mathrm{~b}$ & $68.53 \mathrm{~b}$ & $24.05 \mathrm{e}$ & $73.0 \mathrm{e}$ & $2874.98 \mathrm{c}$ \\
\hline 9 & $36.67 \mathrm{c}$ & $18.17 \mathrm{~b}$ & $51.07 \mathrm{c}$ & $10.78 \mathrm{~b}$ & $22.07 \mathrm{a}$ & $5.73 \mathrm{a}$ & $91.03 \mathrm{a}$ & $18.14 \mathrm{~g}$ & $73.0 \mathrm{e}$ & $2505.90 \mathrm{c}$ \\
\hline 10 & $39.00 \mathrm{a}$ & $20.97 \mathrm{~b}$ & $41.37 \mathrm{~d}$ & $9.72 \mathrm{c}$ & $17.00 \mathrm{~b}$ & $5.60 \mathrm{a}$ & $73.50 \mathrm{~b}$ & $19.51 \mathrm{~g}$ & $87.7 \mathrm{c}$ & $2350.85 \mathrm{c}$ \\
\hline 11 & $35.33 \mathrm{c}$ & $16.70 \mathrm{~b}$ & $52.43 \mathrm{~b}$ & $9.31 \mathrm{c}$ & $24.30 \mathrm{a}$ & $5.17 \mathrm{~b}$ & $94.83 \mathrm{a}$ & $18.59 \mathrm{~g}$ & $73.0 \mathrm{e}$ & $2775.80 \mathrm{c}$ \\
\hline 12 & $36.00 \mathrm{c}$ & $20.67 \mathrm{~b}$ & $57.47 \mathrm{a}$ & $9.97 \mathrm{c}$ & $14.87 \mathrm{~b}$ & $4.90 \mathrm{~b}$ & $57.97 \mathrm{~b}$ & $25.76 \mathrm{e}$ & $79.3 \mathrm{~d}$ & $2103.36 \mathrm{c}$ \\
\hline 13 & $35.33 \mathrm{c}$ & $16.97 \mathrm{~b}$ & $50.30 \mathrm{c}$ & $10.20 \mathrm{c}$ & $19.60 \mathrm{a}$ & $5.30 \mathrm{~b}$ & $70.10 \mathrm{~b}$ & $19.78 \mathrm{~g}$ & $73.0 \mathrm{e}$ & $2139.53 \mathrm{c}$ \\
\hline 14 & $36.33 \mathrm{c}$ & $17.33 \mathrm{~b}$ & $51.90 \mathrm{~b}$ & $11.94 \mathrm{a}$ & $24.07 \mathrm{a}$ & $5.07 \mathrm{~b}$ & $81.83 \mathrm{a}$ & $24.61 \mathrm{e}$ & $73.0 \mathrm{e}$ & $2874.49 \mathrm{c}$ \\
\hline 15 & $40.00 \mathrm{a}$ & $20.17 \mathrm{~b}$ & $49.60 \mathrm{c}$ & $9.82 \mathrm{c}$ & $17.83 \mathrm{~b}$ & $5.17 \mathrm{~b}$ & $75.67 \mathrm{~b}$ & $24.47 \mathrm{e}$ & $85.0 \mathrm{c}$ & $2346.03 \mathrm{c}$ \\
\hline 16 & $33.33 \mathrm{~d}$ & $21.37 \mathrm{~b}$ & $54.60 \mathrm{~b}$ & $10.81 \mathrm{~b}$ & $19.60 \mathrm{a}$ & $4.77 \mathrm{~b}$ & $73.20 \mathrm{~b}$ & $24.77 \mathrm{e}$ & $73.0 \mathrm{e}$ & $3067.62 \mathrm{c}$ \\
\hline 17 & $39.00 \mathrm{a}$ & $29.07 \mathrm{a}$ & $50.50 \mathrm{c}$ & $9.33 \mathrm{c}$ & $14.23 \mathrm{~b}$ & $5.03 \mathrm{~b}$ & $51.20 \mathrm{~b}$ & $24.52 \mathrm{e}$ & $84.3 \mathrm{c}$ & $2006.32 \mathrm{c}$ \\
\hline 18 & $36.00 \mathrm{c}$ & $18.03 \mathrm{~b}$ & $52.47 \mathrm{~b}$ & $10.65 \mathrm{~b}$ & $22.17 \mathrm{a}$ & $6.13 \mathrm{a}$ & $104.70 \mathrm{a}$ & $20.46 \mathrm{f}$ & $74.3 \mathrm{e}$ & $2590.02 \mathrm{c}$ \\
\hline 19 & $38.67 \mathrm{a}$ & $20.70 \mathrm{~b}$ & $40.53 \mathrm{~d}$ & $11.31 \mathrm{a}$ & $18.67 \mathrm{a}$ & $5.93 \mathrm{a}$ & $72.27 \mathrm{~b}$ & $21.28 \mathrm{f}$ & $90.3 \mathrm{~b}$ & $2553.48 \mathrm{c}$ \\
\hline 20 & $34.00 \mathrm{~d}$ & $17.93 \mathrm{~b}$ & $53.47 \mathrm{~b}$ & $10.73 \mathrm{~b}$ & $16.73 b$ & $4.43 \mathrm{~b}$ & $41.90 \mathrm{~b}$ & $43.07 \mathrm{a}$ & $85.3 \mathrm{c}$ & $2723.88 \mathrm{c}$ \\
\hline 21 & $38.00 \mathrm{~b}$ & $23.30 \mathrm{a}$ & $56.13 \mathrm{~b}$ & $10.84 \mathrm{~b}$ & $16.37 \mathrm{~b}$ & $5.13 \mathrm{~b}$ & $64.40 \mathrm{~b}$ & $25.36 \mathrm{e}$ & $82.7 \mathrm{~d}$ & $2636.82 \mathrm{c}$ \\
\hline 22 & $39.00 \mathrm{a}$ & $22.77 \mathrm{a}$ & $52.13 \mathrm{~b}$ & $10.44 \mathrm{c}$ & $16.37 \mathrm{~b}$ & $4.93 \mathrm{~b}$ & $63.53 \mathrm{~b}$ & $24.86 \mathrm{e}$ & $82.0 \mathrm{~d}$ & $2398.31 \mathrm{c}$ \\
\hline 23 & $36.33 \mathrm{c}$ & $22.33 \mathrm{a}$ & $57.53 \mathrm{a}$ & $11.28 \mathrm{a}$ & $15.43 \mathrm{~b}$ & $6.10 \mathrm{a}$ & $70.95 \mathrm{~b}$ & $22.99 \mathrm{e}$ & $75.7 \mathrm{e}$ & $2325.09 \mathrm{c}$ \\
\hline 24 & $39.00 \mathrm{a}$ & $18.03 \mathrm{~b}$ & $48.97 \mathrm{c}$ & $10.41 \mathrm{c}$ & $18.03 \mathrm{~b}$ & $5.67 \mathrm{a}$ & $80.63 \mathrm{a}$ & $25.85 \mathrm{e}$ & $86.7 \mathrm{c}$ & $3178.76 \mathrm{c}$ \\
\hline 25 & $37.00 \mathrm{c}$ & $19.60 \mathrm{~b}$ & $51.43 \mathrm{~b}$ & $10.55 \mathrm{~b}$ & $19.57 \mathrm{a}$ & $5.60 \mathrm{a}$ & $84.37 \mathrm{a}$ & $25.56 \mathrm{e}$ & $75.7 \mathrm{e}$ & $2978.99 \mathrm{c}$ \\
\hline 26 & $35.67 \mathrm{c}$ & $17.13 \mathrm{~b}$ & $46.73 \mathrm{c}$ & $10.00 \mathrm{c}$ & $21.03 \mathrm{a}$ & $5.17 \mathrm{~b}$ & $70.87 \mathrm{~b}$ & $19.91 \mathrm{~g}$ & $74.3 \mathrm{e}$ & $2109.68 \mathrm{c}$ \\
\hline 27 & $34.33 \mathrm{~d}$ & $19.83 \mathrm{~b}$ & $50.97 \mathrm{c}$ & $10.41 \mathrm{c}$ & $17.90 \mathrm{~b}$ & $5.33 \mathrm{~b}$ & $72.97 \mathrm{~b}$ & $25.41 \mathrm{e}$ & $73.0 \mathrm{e}$ & $2862.57 \mathrm{c}$ \\
\hline 28 & $36.33 \mathrm{c}$ & $16.60 \mathrm{~b}$ & $42.80 \mathrm{~d}$ & $9.80 \mathrm{c}$ & $20.70 \mathrm{a}$ & $5.10 \mathrm{~b}$ & $69.67 \mathrm{~b}$ & $20.58 \mathrm{f}$ & $74.3 \mathrm{e}$ & $2040.55 \mathrm{c}$ \\
\hline 29 & $38.00 \mathrm{~b}$ & $20.33 b$ & $49.77 \mathrm{c}$ & $11.68 \mathrm{a}$ & $12.23 \mathrm{~b}$ & $5.50 \mathrm{a}$ & $52.00 \mathrm{~b}$ & $23.72 \mathrm{e}$ & $82.0 \mathrm{~d}$ & $2711.83 \mathrm{c}$ \\
\hline 30 & $35.67 \mathrm{c}$ & $16.40 \mathrm{~b}$ & $47.57 \mathrm{c}$ & $9.75 \mathrm{c}$ & $19.00 \mathrm{a}$ & $4.63 \mathrm{~b}$ & $61.13 \mathrm{~b}$ & $21.66 \mathrm{f}$ & $75.7 \mathrm{e}$ & $1964.78 \mathrm{c}$ \\
\hline 31 & $35.67 \mathrm{c}$ & $20.37 \mathrm{~b}$ & $55.60 \mathrm{~b}$ & $10.55 \mathrm{~b}$ & $23.33 \mathrm{a}$ & $5.50 \mathrm{a}$ & $96.30 \mathrm{a}$ & $20.74 \mathrm{f}$ & $78.0 \mathrm{e}$ & $2897.84 \mathrm{c}$ \\
\hline 32 & $35.33 \mathrm{c}$ & $23.67 \mathrm{a}$ & $50.80 \mathrm{c}$ & $10.04 \mathrm{c}$ & $17.63 \mathrm{~b}$ & $5.17 \mathrm{~b}$ & $64.67 \mathrm{~b}$ & $20.67 \mathrm{f}$ & $73.0 \mathrm{e}$ & $2098.43 \mathrm{c}$ \\
\hline 33 & $36.67 \mathrm{c}$ & $17.43 \mathrm{~b}$ & $49.83 \mathrm{c}$ & $10.59 \mathrm{~b}$ & $16.70 \mathrm{~b}$ & $5.60 \mathrm{a}$ & $66.70 \mathrm{~b}$ & $25.09 \mathrm{e}$ & $75.7 \mathrm{e}$ & $2716.85 \mathrm{c}$ \\
\hline 34 & $35.67 \mathrm{c}$ & $18.07 \mathrm{~b}$ & $49.67 \mathrm{c}$ & $10.00 \mathrm{c}$ & $19.63 \mathrm{a}$ & $5.83 \mathrm{a}$ & $90.40 \mathrm{a}$ & $22.02 \mathrm{f}$ & $80.3 \mathrm{~d}$ & $3112.54 \mathrm{c}$ \\
\hline 35 & $35.33 \mathrm{c}$ & $19.23 \mathrm{~b}$ & $55.47 \mathrm{~b}$ & $11.24 \mathrm{a}$ & $17.00 \mathrm{~b}$ & $4.73 \mathrm{~b}$ & $55.30 \mathrm{~b}$ & $37.88 \mathrm{~b}$ & $79.3 \mathrm{~d}$ & $3101.86 \mathrm{c}$ \\
\hline 36 & $38.00 \mathrm{~b}$ & $22.00 \mathrm{a}$ & $43.17 \mathrm{~d}$ & $9.63 \mathrm{c}$ & $9.57 \mathrm{~b}$ & $5.23 \mathrm{~b}$ & $37.20 \mathrm{~b}$ & $22.02 \mathrm{f}$ & $92.0 \mathrm{~b}$ & $1473.47 \mathrm{c}$ \\
\hline 37 & $38.00 \mathrm{~b}$ & $18.53 \mathrm{~b}$ & $48.23 \mathrm{c}$ & $9.57 \mathrm{c}$ & $14.03 \mathrm{~b}$ & $5.90 \mathrm{a}$ & $61.97 \mathrm{~b}$ & $23.43 \mathrm{e}$ & $82.7 \mathrm{~d}$ & $2828.83 \mathrm{c}$ \\
\hline 38 & $37.33 \mathrm{~b}$ & $20.27 \mathrm{~b}$ & $51.83 \mathrm{~b}$ & $10.04 \mathrm{c}$ & $12.80 \mathrm{~b}$ & $5.73 \mathrm{a}$ & $64.57 \mathrm{~b}$ & $22.91 \mathrm{e}$ & $81.7 \mathrm{~d}$ & $2336.76 \mathrm{c}$ \\
\hline 39 & $38.67 \mathrm{a}$ & $17.03 \mathrm{~b}$ & $46.90 \mathrm{c}$ & $11.27 \mathrm{a}$ & $17.43 \mathrm{~b}$ & $6.53 \mathrm{a}$ & $86.33 \mathrm{a}$ & $25.39 \mathrm{e}$ & $79.3 \mathrm{~d}$ & $3231.34 \mathrm{c}$ \\
\hline 40 & $39.00 \mathrm{a}$ & $20.20 \mathrm{~b}$ & $48.70 \mathrm{c}$ & $10.33 \mathrm{c}$ & $16.17 \mathrm{~b}$ & $6.40 \mathrm{a}$ & $78.93 \mathrm{a}$ & $24.13 \mathrm{e}$ & $81.7 \mathrm{~d}$ & $2516.29 \mathrm{c}$ \\
\hline 41 & $39.00 \mathrm{a}$ & $25.57 \mathrm{a}$ & $56.17 b$ & $9.92 \mathrm{c}$ & $19.43 \mathrm{a}$ & $5.10 \mathrm{~b}$ & $69.83 \mathrm{~b}$ & $28.75 \mathrm{~d}$ & $100.7 \mathrm{a}$ & $3567.60 \mathrm{~b}$ \\
\hline 42 & $39.67 \mathrm{a}$ & $24.87 \mathrm{a}$ & $58.63 \mathrm{a}$ & $10.49 \mathrm{c}$ & $15.97 \mathrm{~b}$ & $5.37 \mathrm{~b}$ & $59.43 \mathrm{~b}$ & $29.90 \mathrm{~d}$ & $99.0 \mathrm{a}$ & $4013.25 \mathrm{~b}$ \\
\hline 43 & $39.67 \mathrm{a}$ & $24.90 \mathrm{a}$ & $59.53 \mathrm{a}$ & $10.32 \mathrm{c}$ & $20.73 \mathrm{a}$ & $6.07 \mathrm{a}$ & $99.03 \mathrm{a}$ & $28.03 \mathrm{~d}$ & $100.7 \mathrm{a}$ & $5347.52 \mathrm{a}$ \\
\hline 44 & $38.00 \mathrm{~b}$ & $22.63 \mathrm{a}$ & $53.07 \mathrm{~b}$ & $10.10 \mathrm{c}$ & $20.20 \mathrm{a}$ & $5.87 \mathrm{a}$ & $90.77 \mathrm{a}$ & $26.36 \mathrm{e}$ & $99.0 \mathrm{a}$ & $4657.80 \mathrm{a}$ \\
\hline 45 & $39.33 \mathrm{a}$ & $27.80 \mathrm{a}$ & $58.90 \mathrm{a}$ & $10.29 \mathrm{c}$ & $17.47 \mathrm{~b}$ & $5.10 \mathrm{~b}$ & $63.53 \mathrm{~b}$ & $28.75 \mathrm{~d}$ & $100.7 \mathrm{a}$ & $3753.08 \mathrm{~b}$ \\
\hline 46 & $39.00 \mathrm{a}$ & $20.93 \mathrm{~b}$ & $52.30 \mathrm{~b}$ & $10.34 \mathrm{c}$ & $19.57 \mathrm{a}$ & $4.80 \mathrm{~b}$ & $66.80 \mathrm{~b}$ & $25.80 \mathrm{e}$ & $100.7 \mathrm{a}$ & $3740.12 \mathrm{~b}$ \\
\hline 47 & $39.00 \mathrm{a}$ & $23.97 \mathrm{a}$ & $55.37 \mathrm{~b}$ & $10.03 \mathrm{c}$ & $26.10 \mathrm{a}$ & $5.50 \mathrm{a}$ & 89.77 a & $28.93 \mathrm{~d}$ & $99.0 \mathrm{a}$ & $5221.27 \mathrm{a}$ \\
\hline 48 & $39.00 \mathrm{a}$ & $24.37 \mathrm{a}$ & $54.60 \mathrm{~b}$ & $9.75 \mathrm{c}$ & $16.73 \mathrm{~b}$ & $5.43 \mathrm{a}$ & $68.33 \mathrm{~b}$ & $23.92 \mathrm{e}$ & $99.0 \mathrm{a}$ & $3718.93 \mathrm{~b}$ \\
\hline 49 & $39.67 \mathrm{a}$ & $25.30 \mathrm{a}$ & $53.80 \mathrm{~b}$ & $10.88 \mathrm{~b}$ & $17.47 \mathrm{~b}$ & $4.97 \mathrm{~b}$ & $67.20 \mathrm{~b}$ & $27.01 \mathrm{e}$ & $99.0 \mathrm{a}$ & $3837.44 \mathrm{~b}$ \\
\hline 50 & $38.00 \mathrm{~b}$ & $20.97 \mathrm{~b}$ & $54.23 \mathrm{~b}$ & $10.21 \mathrm{c}$ & $23.67 \mathrm{a}$ & $5.70 \mathrm{a}$ & $101.20 \mathrm{a}$ & $30.03 \mathrm{~d}$ & $99.0 \mathrm{a}$ & $5743.19 \mathrm{a}$ \\
\hline 51 & $40.33 \mathrm{a}$ & $23.50 \mathrm{a}$ & $51.60 \mathrm{~b}$ & $10.42 \mathrm{c}$ & $16.20 \mathrm{~b}$ & $5.37 \mathrm{~b}$ & $71.93 \mathrm{~b}$ & $31.24 \mathrm{c}$ & $99.0 \mathrm{a}$ & $4623.30 \mathrm{a}$ \\
\hline 52 & $39.00 \mathrm{a}$ & $26.90 \mathrm{a}$ & $52.77 \mathrm{~b}$ & $9.93 \mathrm{c}$ & $14.97 \mathrm{~b}$ & $6.03 \mathrm{a}$ & $65.30 \mathrm{~b}$ & $29.69 \mathrm{~d}$ & $99.0 \mathrm{a}$ & $3577.75 \mathrm{~b}$ \\
\hline 53 & $39.00 \mathrm{a}$ & $22.67 \mathrm{a}$ & $52.63 \mathrm{~b}$ & $9.45 \mathrm{c}$ & $19.23 \mathrm{a}$ & $5.77 \mathrm{a}$ & $68.63 \mathrm{~b}$ & $26.52 \mathrm{e}$ & $99.0 \mathrm{a}$ & $4026.13 \mathrm{~b}$ \\
\hline 54 & $39.33 \mathrm{a}$ & $23.70 \mathrm{a}$ & $58.20 \mathrm{a}$ & $9.38 \mathrm{c}$ & $15.53 \mathrm{~b}$ & $4.97 \mathrm{~b}$ & $61.90 \mathrm{~b}$ & $25.48 \mathrm{e}$ & $99.0 \mathrm{a}$ & $3566.85 \mathrm{~b}$ \\
\hline 55 & $39.67 \mathrm{a}$ & $25.53 \mathrm{a}$ & $62.27 \mathrm{a}$ & $10.12 \mathrm{c}$ & $16.33 \mathrm{~b}$ & $5.13 \mathrm{~b}$ & $58.63 \mathrm{~b}$ & $24.35 \mathrm{e}$ & $99.0 \mathrm{a}$ & $2798.52 \mathrm{c}$ \\
\hline 56 & $39.00 \mathrm{a}$ & $26.87 \mathrm{a}$ & $61.80 \mathrm{a}$ & $10.86 \mathrm{~b}$ & $17.13 \mathrm{~b}$ & $5.90 \mathrm{a}$ & $73.13 \mathrm{~b}$ & $25.72 \mathrm{e}$ & $100.7 \mathrm{a}$ & $3337.15 \mathrm{c}$ \\
\hline 57 & $31.00 \mathrm{e}$ & $21.90 \mathrm{a}$ & $46.73 \mathrm{c}$ & $9.98 \mathrm{c}$ & $14.70 \mathrm{~b}$ & $5.07 \mathrm{~b}$ & $54.50 \mathrm{~b}$ & $31.44 \mathrm{c}$ & $82.0 \mathrm{~d}$ & $3065.59 \mathrm{c}$ \\
\hline 58 & $34.00 \mathrm{~d}$ & $26.63 \mathrm{a}$ & $41.13 \mathrm{~d}$ & $9.09 \mathrm{c}$ & $13.47 \mathrm{~b}$ & $4.83 \mathrm{~b}$ & $49.00 \mathrm{~b}$ & $35.79 \mathrm{~b}$ & $82.0 \mathrm{~d}$ & $3083.35 \mathrm{c}$ \\
\hline
\end{tabular}

Genetics and Molecular Research 18 (2): gmr18299 
This characteristic has importance in the grains quality in relation to the first pod height insertion, that is, long pods inserted closer to the soil increase the possibility of diseases in the pods together with reduction in grain quality. Kappes et al. (2008) determined that a first pod insertion height of $15.5 \mathrm{~cm}$ along with the pod length of 6.4 are favorable to mechanized harvesting, guaranteeing grain quality and lower crop loss.

For ANPP, ANSP and ANSPL there were two distinct groups, ranging from 9.57 to 26.10 pods per plant, from 4.07 to 6.53 seeds per pods and from 37.2 to 104.7 seeds per plant. Correa and Gonçalves (2012), when analyzing the genetic divergence among 13 genotypes, between cultivars and lines of common bean, obtained results similar to those observed for these characteristics, with number of pods per plant slightly above those found, the observed values are concordant with common bean culture and because they are factors of production can be considered positive at the moment of selection of parents for hybridization and obtaining upper segregants.

For W100 there were seven groups, ranging from 17.21 to 43.07 grams. For the GPROD variable, three groups were formed, and the improved genotypes were responsible for the best performance, group I, with yields more than $3.566 .85 \mathrm{Kg}_{\text {.ha }}{ }^{-1}$. The other genotypes were allocated to groups II and III constituents of traditional accessions and only four improved genotypes, with GPROD ranging from 1.473 .47 to $3.337 .15 \mathrm{Kg}_{\text {.ha }}{ }^{-1}$, which shows a superior productive performance of improved genotypes in relation to traditional accesses, however, when comparing this performance of groups II and III with the national average of common bean productivity that is $1.004 .00 \mathrm{~kg} \cdot \mathrm{ha}^{-1}$, we observe that we have favorable results with potential to be explored in all genotypes studied.

Oliveira et al. (2012), evaluating 11 cultivars of common bean obtained similar results for W100, presenting the formation of only three groups, but presented similar weight to those obtained (ranging from 17.15 to $30.00 \mathrm{~g}$ ). Farinelli and Lemos (2010), reaching yields higher than $4.157 .00 \mathrm{~kg} \cdot \mathrm{ha}^{-1}$ when evaluating agronomic characteristics and productive performance of 24 genotypes of common bean (carioca and black commercial group), confirming the fact that the improved genotypes present higher yields of grains through the beneficial effect of yield components such as number of pods per plant and mass of 100 grains.

The observed results favor the use of these genotypes to compose the breeding program in question since the studied variables demonstrate the existence of genotypes with favorable alleles that will provide a positive increase in the production components and that will directly influence the yield of grains.

The estimated divergence among genotypes, based on the generalized distance of Mahalanobis $\left(D_{i i^{\prime}}^{2}\right)$, made it possible to identify the combination with greater magnitude of dissimilarity between genotypes 4 (Mesoamerican) and 20 (Andean), $\left(D_{i i^{\prime}}^{2}=346.01\right.$ ), and based on the genetic distance this would be the most promising crossing for maximum heterosis. Other combinations with high magnitude dissimilarity were found between genotype 11 and $20\left(D_{i i^{\prime}}^{2}=332.88\right), 3$ and $55\left(D_{i i^{\prime}}^{2}=341.72\right)$ and 3 with $43\left(D_{i i^{\prime}}^{2}=\right.$ 330.62). The lowest magnitude of dissimilarity was between genotypes 13 and 26 because they had a minimum value of $D_{i i^{\prime}}^{2}=1.51$, both belonging to the Mulatinho group, determining these accessions as the most similar among the others, not being recommended for crosses between them. According to Singh (2001) knowledge of genetic diversity makes 
it possible to improve efficiency in the conservation, utilization and genetic improvement of common bean.

In the cluster analysis of the genotypes by the Tocher optimization method, we have the formation of six distinct groups, allocating the most similar genotypes within the different groups (Table 4). Group I, composed of 22 genotypes, was the most numerous with $37.95 \%$ of the total, where it clustered only traditional genotypes. The group II, consisting of 20 genotypes, with $34.48 \%$ of all genotypes evaluated, of which only 4 are traditional genotypes and the others are improved genotypes. Group III was composed of 11 genotypes (18.96\%), consisting only of traditional genotypes. The group IV and V, formed by only two genotypes each, conferring a percentage of 18.95 of the total genotypes studied, allocating only genotypes of the Andean gene group, except for genotype 57, being Mesoamerican. Finally, group VI, containing only the genotype $58(1.72 \%$ of the total studied) belonging to the Andean gene group, being a genotype of the commercial group Enxofre.

Table 4. Representation of the clusters of genotypes generated by the Tocher optimization method based on the dissimilarity among the 58 accessions of common bean.

\begin{tabular}{llr}
\hline Groups & Accesses & \% of accesses \\
\hline I & $13,26,30,28,2,32,9,31,18,11,4,34,23,27,33,25,12,16,38,1,8$ and 14 & 37.93 \\
II & $41,45,42,49,56,46,48,54,55,53,44,43,47,52,50,51,6,7,5$ and 24 & 34.48 \\
III & $21,22,29,19,15,14,40,37,39,17,10$ and 36 & 20.69 \\
IV & 3 and 57 & 3.44 \\
V & 20 and 35 & 3.44 \\
VI & 58 & 1.72 \\
Total & 58 & 100.0 \\
\hline
\end{tabular}

The groups showed a clear division of the traditional accessions to the cultivars and improved lines, besides separating the genotypes of Andean of the genotypes of Mesoamerican origin. This distribution of genotypes in relation to the center of origin was also reported by Cabral et al. (2018) investigating the genetic diversity of 57 common bean accessions.

Additionally Cabral et al. (2011), evaluating the genetic divergence in 57 common bean genotypes shows that most of the improved genotypes are clustered in only one group, and Rodrigues et al. (2002), with the objective of determining the genetic diversity of common bean cultivars in Rio Grande do Sul state, using agronomic descriptors, reported that the commercial genotypes evaluated presented a high degree of similarity between them, so these studies corroborate with the present research considering that in group II we have the union of $90 \%$ of the improved genotypes, that is, among them the variability is low in relation to the traditional accesses.

The low diversity presented in the cultivars is linked to the center of origin and the domestication of the common bean culture, since, except for the line TB03-13, that has Andean origin, all the others have Mesoamerican origin, and as pointed out by Schmutz et al. (2014), in the process of domestication the reduction of diversity was more pronounced in the Mesoamerican races.

The largest intragroup distance was observed in group $I\left(d_{I}=26.20\right)$ and the lowest in group IV $\left(d_{I}=19.14\right)$, where group $I$, besides being the largest group, is the group that 
has the greatest genetic variability within the group in front of the other groups formed (Table 5). Likewise, the greatest intergroup distance was between groups II and $\mathrm{V}\left(\mathrm{d}_{\mathrm{I}}=\right.$ 235.37), suggesting that crosses between genotypes of these two groups tend to produce progenies with a broader genetic base with favorable alleles and potential to be exploited in the breeding program. According to Gonçalves et al. (2014), through the intergroup analysis we showed the most divergent genotypes, suggesting that we cross between groups with higher intergroup distance values.

Table 5. Average intra and intergroup distance estimated by the Tocher optimization method based on the dissimilarity among the 58 accessions of common bean.

\begin{tabular}{llrrrrr}
\hline & I & II & III & IV & V & VI \\
\hline I & 26.20 & 126.34 & 59.36 & 110.07 & 186.91 & 145.38 \\
II & 25.18 & 55.52 & 235.37 & 188.38 & 171.23 \\
III & & 25.76 & 167.76 & 182.94 & 135.47 \\
IV & & & 19.14 & 90.80 & 52.72 \\
V & & & & 20.35 & 71.32 \\
VI & & & & & - \\
\hline
\end{tabular}

Between groups IV and VI, we have the smallest intergroup distance $\left(d_{I}=52.72\right)$, determining that the cross between genotypes of these groups should be avoided, since it presents difficulties to generate genotypes with potential superior to the parents, since the parents have a lot of proximity to the characteristics. According to Vasconcelos et al. (2007), there will always be more homogeneity between genotypes of the same group than between genotypes of different groups, since the Tocher method presents the average distance within the groups always smaller than the average distance between the groups.

When analyzing the dendrogram of the Hierarchical UPGMA method (Figure 1), we can visualize the formation of 5 distinct groups, clustering the genotypes based on the agronomic characteristics analyzed, providing information to choose possible genotypes to be selected to form a cross-scheme, thus allowing the generation of transgressive segregants.

The 22 genotypes belonging to Group I $(13,26,28,30,32,11,4,9,18,34,31,16$, $27,8,14,1,25,33,12,23$ e 39) presented as main characteristics for the formation of this group, greater number of pods per plant and number of seeds per plant, combined with lower mass of 100 seeds and earlier genotypes. Group III, constituted by 15 genotypes (19, $29,10,36,37,38,5,24,40,15,21,22,6,17$ e 7 ) has the main characteristic of this group being a higher average number of seeds per pods, but in compensation they presented lower grain yield. Both groups were formed by genotypes belonging to the Mesoamerican gene group and constituted only by traditional accesses.

The Group II, composed of the accessions 51, 52, 44, 53, 47, 50, 43, 41, 45, 42, 46, $49,48,54,55$ e 56 , has a characteristic of the genotypes that compose this group, the later cycle, in addition to presenting greater pod insertion, higher plant height and higher grain yield, since only promising cultivars or lines were allocated in this group. Group IV, constituted by accessions 20 and 35, having the main characteristics of the group, due to the greater length of pod and mass of 100 seeds, and lower height of pod insertion, lower number of seeds per pod and seeds per plant. Group V, formed by accessions 3, 57 and 58, 
had the shortest period of flowering, lower plant height, lower pod length and lower number of pods per plant, thus ensuring the grouping of these accessions in this group.

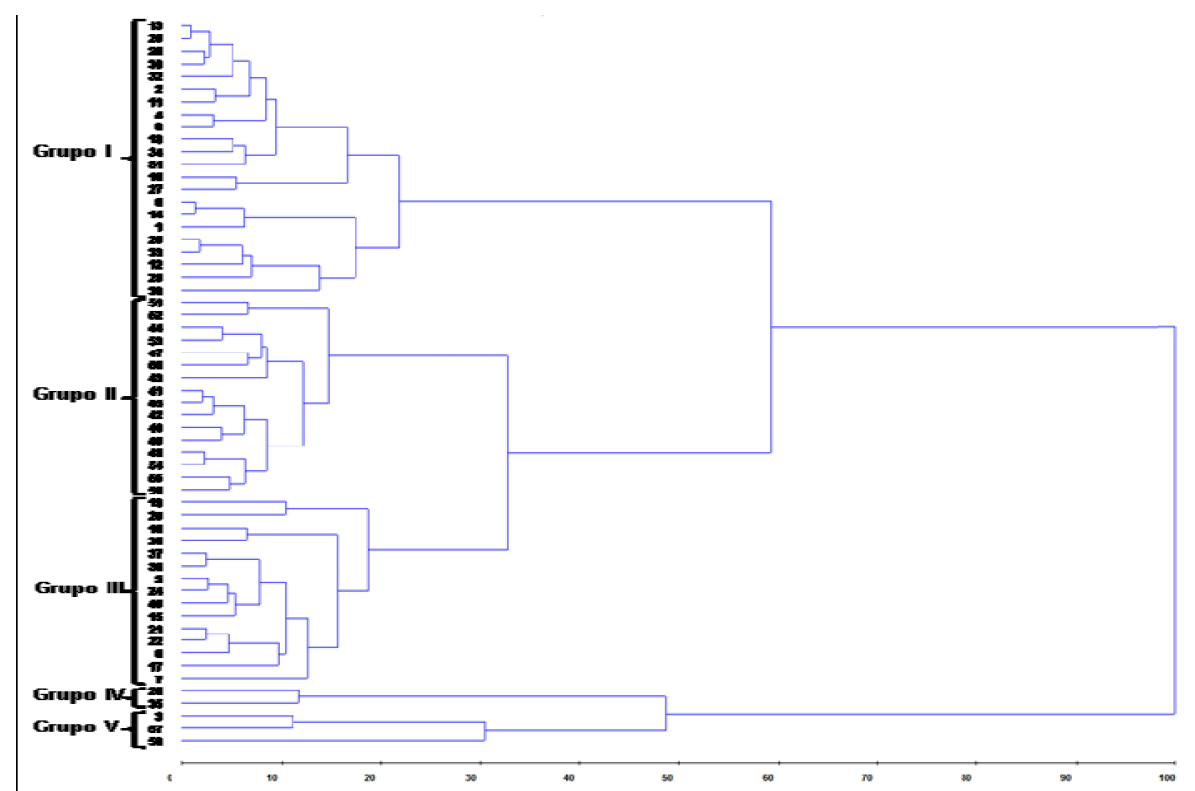

Figure 1. Dendrogram generated by the hierarchical method of UPGMA, and the respective groups, based on the generalized distance of Mahalanobis of 58 common bean genotypes evaluated in Cáceres, MT.

The UPGMA method showed similarity in the grouping of the evaluated genotypes, ordering the most divergent genotypes in different groups and the most similar ones in the same group, besides similarly separating the improved genotypes from the traditional accesses, evidencing the existence of genetic variability both between traditional and improved genotypes, as well as between traditional and improved genotypes. In addition, it showed similarity to the groupings generated by the Tocher method, where group I and III, composed only of traditional genotypes, group II separated only the cultivars and promising lines by the UPGMA method and allocated more accessions 5, 6, 7 and 24 in the Tocher, being identical for both grouping methods. On the other hand, the Tocher group IV, V and VI, formed by genotype of the Andean gene group, separated the genotypes in only two groups in the UPGMA.

Studies carried out by Gonçalves et al. (2014), evaluating the genetic divergence of 65 traditional accessions of common bean, through the clustering method of UPGMA, allowed the formation of VI groups, being efficient in determining the existence of genetic variability among the evaluated accesses, besides being concordant in the form of clustering of accessions when compared with the Tocher method.

Several authors studying the genetic divergence between improved genotypes and traditional accesses, obtained results that demonstrate the similarity between the UPGMA and Tocher methodologies, in agreement with the work in question, the authors also observe a joining of some genotypes and reduction of groups in UPGMA (Bonett, et al., 2006; Elias et al., 2007; Barelli et al., 2009; Bertini et al., 2009; Bertini et al., 2010). 
Thus, the genetic divergence observed between the groups formed by the two Tocher and UPGMA methodologies allows us to select improved parents with positive characteristics for productivity and mechanized harvest, with traditional accessions that present favorable alleles for precocity, that can be crossed and thus obtain transgressive segregants for the characteristics of precocity and productivity, which allow us to initiate a common bean genetic improvement program.

\section{CONCLUSIONS}

The genotypes presented significant genetic variability, indicating that they are promising for exploitation in a breeding program, especially the genotypes: 11 (Manteigão), 13 (Mulatinho) and 16 (Carioca), which are earlier, besides presenting greater dissimilarity when compared with genotypes 43 (IPR Uirapuru) and 51 (FT 08-47), and are highly productive, being useful therefore for initiating a common bean breeding program.

\section{ACKNOWLEDGMENTS}

The authors are grateful to Fundação de Amparo à Pesquisa do Estado de Mato Grosso (FAPEMAT) for granting a scholarship.

\section{CONFLICTS OF INTEREST}

The authors declare no conflict of interest.

\section{REFERENCES}

Alvares RC, Reis EF and Pinto JFN (2012). Divergência genética entre genótipos de pimenta coletados no sudoeste goiano. Ciênc. Agrotec. 5: 498-506.

Arantes EM, Cremon C and Luiz MAC (2012). Alterações dos atributos químicos do solo cultivado no sistema orgânico com plantio direto sob diferentes coberturas vegetais. Rev. Agrarian. 5: 47-54.

Barelli MAA, Gonçalves-Vidigal MC, Vidigal-Filho PS, Neves LG, et al. (2009). Genetic divergence in common bean landrace cultivars from Mato Grosso do Sul State. Semina. 30: 1061-1072. https://doi.org/10.5433/16790359.2009v30n4Sup1p1061

Beraldo ALA, Colombo CA, Chiorato AF, Ito MF, et al. (2009). Aplicação de marcadores scars para a seleção de linhagens resistentes à antracnose em feijoeiro. Bragantia. 68: 53-61. http://dx.doi.org/10.1590/S000687052009000100007.

Bertini CHCM, Almeida WS, Silva APM, Lima JW, et al. (2010). Análise multivariada e índice de seleção na identificação de genótipos superiores de feijão-caupi. Acta Sci. Agron. 32: 613-619. https://doi.org/ 10.4025/actasciagron.v32i4.4631.

Bertini CHCM, Teófilo EM, Dias FTC (2009). Genetic divergence among cowpea UFC germplasm bank accessions. Rev. Ciênc. Agron. 40:99-105.

Bertoldo JG, Coimbra JLM, Guidolin AF, Andrade LRB, et al. (2014). Agronomic potential of genebank landrace elite accessions for common bean. Sci. Agri. 71: 120-125. http://dx.doi.org/10.1590/S0103-90162014000200005.

Bonett LP, Gonçalves-Vidigal MC, Schuelter AR, Vidigal-Filho OS, et al. (2006). Genetic divergence in germplasm of common bean in Paraná State, Brazil. Semina. 27: 547-560. http://dx.doi.org/10.5433/1679-0359.2006v27n4p547.

Broughton WJ, Hernández G, Blair MW, Beebe SE, et al. (2003). Beans (Phaseolus vulgaris L, spp,) - model food legumes. Plant Soil. 252: 55-128. http://dx.doi.org/10.1023/A:1024146710611.

Buratto JS, Moda-Cirino V, Júnior NSF, Prete CEC, et al. (2007). Agronomic performance and grain yield in early common bean genotypes in Paraná state. Semina. 28: 373-380. http://dx.doi.org/10.5433/16790359.2007v28n3p373.

Burle ML, Fonseca JR, Kami JA and Gepts P (2010). Microsatellite diversity and genetic structure among common bean (Phaseolus vulgaris L.) landraces in Brazil, a secondary center of diversity. Theor. Appl. Genet. 121: 801-813. http://dx.doi.org/10.1007/s00122-010-1350-5. 
Cabral PDS, Soares TCB, Lima ABP, Alves DS, et al. (2011). Genetic diversity of common bean accessions by agronomic traits. Rev. Ciênc. Agron. 42: 898-905. http://dx.doi.org/10.1590/S1806-66902011000400011.

Cabral PDS, Souza, LC de, Costa GF da, Silva FHL, et al. (2018). Investigation of the genetic diversity of common bean (Phaseolus vulgaris) cultivars using molecular markers. Genet. Mol. Res. 17: 1-11. https://doi.org/10.4238/gmr18106.

Coelho CM, Coimbra JLM, Souza CA, Bogo A, et al. (2007). Diversidade genética em acessos de feijão (Phaseolus vulgaris L.). Ciênc. Rural. 37: 1241-1247. https://doi.org/10.1590/S0103-84782007000500004.

CONAB. Compania Nacional de Abastecimento. Acompanhamento de safra brasileira: grãos, 2018/19. Available at: <//http://www.conab.gov.br/OlalaCMS/uploads/arquivos/16_01_12_09_00_46_boletim_graos_janeiro_2016.pdf> Accessed on: 05 January 2019.

Correa AM and Gonçalves MC (2012). Divergência genética em genótipos de feijão comum cultivados em Mato Grosso do Sul. Rev. Ceres. 59: 206-212. http://dx.doi.org/10.1590/S0034-737X2012000200009.

Cruz CD (2013). GENES - a software package for analysis in experimental statistics and quantitative genetics. Acta Sci. Agron. 35: 271-276. http://dx.doi.org/10.4025/actasciagron.v35i3.21251.

Dallacort R, Neves SMAS and Nunes MCM (2014). Variabilidade da Temperatura e das Chuvas de Cáceres/Pantanal Mato-Grossense - Brasil. Geografia. 23: 21-33.

Elias HT, Gonçalves-Vidigal MC, Gonela A and Vogt GA (2007). Variabilidade genética em germoplasma tradicional de feijão-preto em Santa Catarina. Pesq. Agropec. Bras. 42: 1443-1449. http://dx.doi.org/10.1590/S0100204X2007001000011.

FAO (2019). Faostat database gateway, Food and Agricultural commodities production. Available AT: <http://faostat,fao,org/site/339/default,aspx>. Accessed on: 08 January 2019.

Farinelli R and Lemos LB (2010). Características agronômicas de genótipos de feijoeiro cultivados nas épocas da seca e das águas. Bragantia. 69: 361-366. http://dx.doi.org/10.1590/S0006-87052010000200013.

Ferreira Junior JA, Unêda-Trevisoli SH, Espíndola SMCG, Vianna VF, et al. (2015). Genetic diversity in advanced soybean strains derived from biparental, four-way and eight-way crosses. Rev. Ciênc. Agron. 46: 339-351. http://dx.doi.org/10.5935/1806-6690.20150013.

Gonçalves DL, Ambrozio VC, Barelli MAA, Neves LG, et al. (2014). Divergência genética de acessos tradicionais de feijoeiros através de características da semente. Biosci. J. 30: 1671-1681.

Hoisington D, Khairallah M, Reeves T, Ribaut JM, et al. (1999). Plant genetic resources: What can they contribute toward increased crop productivity?. Proc. Natl. Acad. Sci. USA. 96: 5937-5943. https://doi.org/10.1073/pnas.96.11.5937.

Kappes CE, Wruck FJ, Carvalho MAC and Yamashita OM (2008). Feijão comum: características morfo-agronômicas de cultivares. IAC. 85: 506-509.

Lima MS, Carneiro JES, Carneiro PCS, Pereira CS, et al. (2012). Characterization of genetic variability among common bean genotypes by morphological descriptors. Crop Breed. Appl. Biotechnol. 12: 76-84. http://dx.doi.org/10.1590/S1984-70332012000100010.

Nass LL, Sigrist MS, Ribeiro CSC and Reifschneider FJB (2012). Genetic resources: the basis for sustainable and competitive plant breeding. Crop Breed. Appl. Biotechnol. S2: 75-86. https://dx.doi.org/10.1590/S198470332012000500009 .

Oliveira TC, Silva J, Sousa SA, Campestrini R, et al. (2012). Yield potential of common bean cultivars in function of phosphorus stress in State of Tocantins. J. Biotec. Biodivers. 3: 24-30.

Peluzio JM, Vaz-de-Melo A, Afférri FS, Silva RR, et al. (2009). Variabilidade genética entre cultivares de soja, sob diferentes condições edafoclimáticas. Pesqui. Apl. Agrotec. 2: 21-29.

Ramalho MAP, Silva GS and Dias LAS (2009). Genetic plant improvement and climate changes. Crop Breed. Appl. Biotechno. 9: 189-195.

Rao RC (1952). Advanced statistical methods in biometric research. New York: J. Willey, 390.

Ribeiro ND, Hoffmann-Junior L and Possebon SB (2004). Genetic variability for cycle in black and carioca commercial dry bean groups. CAST. 10: 19-29. http://dx.doi.org/10.18539/cast.v10i1.685.

Rodrigues LS, Antunes IF, Teixeira MG and Silva JB (2002). Divergência genética entre cultivares locais e cultivares melhoradas de feijão. Pesq. Agropec. Bras. 37: 1275-1284. http://dx.doi.org/10.1590/S0100-204X2002000900011.

Salgado FHM, Fidelis RR, Carvalho GL, Santos GR, et al. (2011). Bean genotypes behavior on offseason at south of state of Tocantins. Biosci. J. 27: 52-58.

Singh SP (2001) Broadening the Genetic Base of Common Bean Cultivars. Crop Science. 41: 1659-1675. http://dx.doi.org/10.2135/cropsci2001.1659.

Silva FB, Ramalho MAP and Abreu AFB (2007). Phenotypic recurrent selection for early flowering of 'Carioca' common bean. Pesq. Agropec. Bras. 42: 1437-1442. http://dx.doi.org/10.1590/S0100-204X2007001000010.

Silva GO, Pereira AS, Souza VQ, Carvalho FIF, et al. (2008). Importance of characters in the dissimilarity of potato progênies in early generations. Bragantia. 67: 141-144. http://dx.doi.org/10.1590/S0006- 7052008000100017.

Schmutz J, McClean PE, Mamidi, S, Wu GA, et al. (2014). A reference genome for common bean and genome-wide analysis of dual domestications. Nature Genetics. 46: 707-713. http://dx.doi.org/10.1038/ng.3008. 
Tsutsumi CY, Bulegon JG and Piano JT (2015). Melhoramento genético do feijoeiro: avanços, perspectivas e novos estudos, no âmbito nacional. Nativa. 3: 217-223. http://dx.doi.org/10.14583/2318-7670.v03n03a12.

Vasconcelos ES, Cruz CD, Bhering LL and Resende-Junior MFR (2007). Alternative methodology for the cluster analysis. Pesq. Agropec. Bras. 42: 1421-1428. http://dx.doi.org/10.1590/S0100-204X2007001000008.

Vieira C, Paula-Júnior TJE and Borém A (2006). Feijão, Viçosa: UFV, 600. 